\title{
Heterokaryon Incompatibility and Heterozygous Diploid Production in Tomato Wilt Isolates of Verticillium dahliae
}

\author{
By L. W. O'GARRO AND J. M. CLARKSON*† \\ Department of Biology, University of the West Indies, PO Box 64, Bridgetown, Barbados
}

(Received 24 March 1988; revised 20 June 1988)

\begin{abstract}
Auxotrophs derived by UV or $N$-methyl- $N^{\prime}$-nitro- $N$-nitrosoguanidine mutagenesis from three North American, three European and four Australian tomato wilt isolates of Verticillium dahliae were used to investigate heterokaryon compatibility. Ninety 'crosses' involving race-1 and race2 isolates were attempted using mixed conidial suspensions and a 'hanging drop' technique. Crosses between North American and Australian isolates failed to produce heterokaryons over a $20 \mathrm{~d}$ period but within each of these two groups of isolates (with one exception) the frequency of heterokaryon formation was $100 \%$. In crosses involving the three European isolates the frequency of heterokaryon formation varied from 0 to $100 \%$. Two of the European isolates produced heterokaryons both with North American and with Australian isolates. Prototrophic diploid conidia were recovered from 13 of the 30 crosses that produced heterokaryons. The rate of diploid production was greatest in crosses showing $100 \%$ compatibility but rare diploids were recovered from two crosses which had produced no observable heterokaryotic growth on minimal medium. The formation of heterozygous diploids between race-1 and race- 2 isolates of $V$. dahliae indicates the potential for the genetic analysis of pathogenicity through the parasexual cycle.
\end{abstract}

\section{INTRODUCTION}

Verticillium dahliae Kleb. and Verticillium albo-atrum $\mathbf{R}$. and $\mathbf{B}$. have been recognized as vascular wilt pathogens of major importance in a wide range of economically important crops (Pegg, 1974; Mace et al., 1981). Tomato wilt isolates of $V$. dahliae may be differentiated into two races: race-1 isolates are non-pathogenic towards tomato cultivars possessing the dominant $V e$ resistance gene whereas race-2 isolates may cause severe symptoms in these cultivars (Pegg, 1974; O'Garro \& Clarkson, 1988). Sexual reproduction is unknown in these vascular pathogens but heterokaryosis, diploid formation and parasexual recombination have been demonstrated (Hastie, 1962; Fordyce \& Green, 1964; Typas \& Heale, 1978) and may be important mechanisms for generating variability in the wild. Genetic studies in this genus have been reviewed recently (Hastie \& Heale, 1985; Heale, 1988).

The formation of a heterozygous diploid in a normally haploid fungus requires the establishment of at least a transient heterokaryon and therefore genetic analysis through the parasexual cycle is limited to those strains which are heterokaryon compatible. Studies of heterokaryosis in fungi have utilized both auxotrophic and morphological markers. Using spore colour mutants, Jinks et al. (1966) demonstrated that a complex incompatibility system operates in wild populations of Aspergillus nidulans, which limits heterokaryon establishment. In all cases studied in detail, vegetative or heterokaryon incompatibility has been shown to be due to heterogeneity at specific gene loci. In Neurospora crassa, ten heterokaryon incompatibility loci

\footnotetext{
† Present address: Department of Plant Biology, University of Bath, Bath BA2 7AY, UK.
}

Abbreviation: NTG, $N$-methyl- $N^{\prime}$-nitro- $N$-nitrosoguanidine. 
have been identified (Perkins \& Barry, 1977). Using auxotrophs, Puhalla \& Speith (1983) have suggested that at least four nuclear gene loci control heterokaryon incompatibility in Fusarium moniliforme. Using mutants that were unable to reduce nitrate Puhalla $(1984 a, b, 1985)$ has reported distinct heterokaryon incompatibility groups in the vascular wilt pathogen Fusarium oxysporum. Using auxotrophic mutants of Verticillium, several instances of the failure to establish a heterokaryon have been reported (Heale, 1966; Typas \& Heale, 1976). Based on studies using both microsclerotial colour mutants and auxotrophs, Puhalla (1979) and Puhalla \& Hummel (1983) have classified isolates of $V$. dahliae from different host plants and geographical origins into a number of heterokaryon incompatibility groups.

The main aim of this study was to investigate the genetic basis of pathogenicity in tomato wilt isolates of $V$. dahliae. We report here the extent of heterokaryon incompatibility between race-1 and race- 2 isolates from different geographical origins and the isolation of heterozygous diploid conidia from certain heterokaryons.

\section{METHODS}

Pathogen culture and mutagenesis. The 10 isolates of $V$. dahliae used in this study are shown in Table 1 . All were obtained from tomato plants except isolate 10 which was from eggplant, and all produced typical microsclerotia on minimal medium. Cultures were derived from single spores to provide parent cultures for all further work. Oxoid modified Czapek Dox served as minimal medium (MM). Complete medium (CM) contained the constituents of MM supplemented with $2 \mathrm{~g} \mathrm{l}^{-1}$ of each of the following: casein hydrolysate, yeast extract, malt extract and mycological peptone. Specific nutritional requirements were added to the $\mathrm{MM}$ at $20 \mu \mathrm{g} \mathrm{ml}^{-1}$ for amino acids, purines and pyrimidines and $1 \mu \mathrm{g} \mathrm{ml}^{-1}$ for vitamins. Solid media contained $15 \mathrm{~g} \mathrm{l}^{-1}$ Difco Bacto agar. Glucose MM (GMM) agar consisted of Czapek Dox MM with glucose substituted for sucrose. Autoclaving was at $120^{\circ} \mathrm{C}$ for $20 \mathrm{~min}$. Cultures were routinely maintained at $25^{\circ} \mathrm{C}$ in the dark. Conidial suspensions for mutagenesis were prepared by washing off a CM plate, which had been spread previously with $0.1 \mathrm{ml}$ of conidial suspension and incubated for 7-8 d, with $10 \mathrm{ml}$ sterile distilled water and adjusted to $5 \times 10^{6}$ conidia $\mathrm{ml}^{-1}$. Auxotrophs were isolated after treatment of wild-type isolates with UV irradiation or $N$-methyl- $N^{\prime}$-nitro- $N$-nitrosoguanidine (NTG) as described previously (Clarkson \& Heale, 1985a). The survival rate after either $3 \mathrm{~min}$ UV or $10 \mathrm{~min}$ NTG $\left(150 \mu \mathrm{g} \mathrm{ml}^{-1}\right)$ treatment was $5 \%$. Auxotrophic requirements are abbreviated according to the system proposed by Demerec et al. (1966).

Heterokaryon compatibility testing. The techniques for producing heterokaryons and heterozygous diploids were based on those described by Hastie (1973) and Ingle \& Hastie (1974) and modified by Clarkson \& Heale (1985b). Conidial suspensions were prepared after 3-4 d growth on CM and then mixed conidial droplets were placed on the base of a sterile Petri dish. Moist sterile filter paper was inserted in the lid, and the dishes were inverted and

Table 1. Geographical origin and race designation of the isolates of V. dahliae used in this study

$V$. dahliae
isolate

$2^{a}$
$3^{b}$
$4^{c}$
$5^{d}$
$6^{d}$
$7^{e}$
$8^{e}$
$10^{b}$
$12^{d}$
$13^{d}$

$\begin{aligned} & \text { Original race } \\ & \text { designation }\end{aligned}$
TS-1, race-1
TOM-34, race-1
Swansea, race-1
58 , race-1
$77-10$ c, race 1
105 , race-2
RG, race-2
Eggplant, race-2
616 , race-2
234 , race-2

Origin

$\begin{array}{lc}\text { California (USA) } & - \\ \text { Greece } & - \\ \text { UK } & - \\ \text { Australia } & 1977 \\ \text { Australia } & 1977 \\ \text { N. Carolina (USA) } & 1976 \\ \text { N. Carolina (USA) } & 1981 \\ \text { Greece } & - \\ \text { Australia } & 1982 \\ \text { Australia } & 1979\end{array}$

* The suppliers of the isolates were as follows, $(a)$ W. J. Tolmsoff, National Cotton Pathology Research Laboratory, ARS, USDA, College Station, Texas 77841, USA; (b) E. C. Tjamos, Benaki Phytopathological Institute, Athens, Greece; $(c)$ M. Milton, Department of Microbiology, University of Swansea, Swansea, UK; $(d)$ R. G. O'Brien, Department of Primary Industries, Meirs Road, Indoorpilly, Queensland, Australia; $(e)$ R. G. Gardner, Department of Horticultural Science, Mountain Horticultural Crops Research Station, North Carolina, USA. 
incubated at $25^{\circ} \mathrm{C}$ for $24 \mathrm{~h}$. The resulting mycelial pellets were then washed in sterile distilled water for $1 \mathrm{~h}$ and transferred to GMM at $29^{\circ} \mathrm{C}$. For each cross between complementary monoauxotrophs, 200 mycelial pellets were transferred to GMM, five on each plate. Heterokaryon compatibility between isolates was assessed as the percentage of 100 mixed mycelial pellets which produced prototrophic growth on GMM over a $20 \mathrm{~d}$ period. The remaining 100 pellets were used to assess the production of heterozygous diploid conidia over a $20 \mathrm{~d}$ period. To check for possible reversion to prototrophy, 100 pellets of each parent auxotroph were transferred to GMM, five per plate, and incubated at $29^{\circ} \mathrm{C}$. Crosses in which one or more pellets of either auxotroph alone grew on GMM were discarded. To distinguish if apparent complementation between auxotrophs was due to cross-feeding rather than heterokaryosis, pairings were made in which cellophane was interposed between the mycelial pellets of the two complementary auxotrophs on GMM. Pairings which produced prototrophic growth under these conditions were also discarded.

Isolation of heterozygous prototrophic diploids. To isolate heterozygous diploid conidia, five randomly selected heterokaryons or mixed mycelial pellets were cut out from the GMM agar plates each day and transferred to $5 \mathrm{ml}$ of sterile distilled water. After gentle agitation the total volume was spread onto MM agar plates and incubated at $25^{\circ} \mathrm{C}$. After $5 \mathrm{~d}$ the number of colonies producing conidia in the diploid size range $(8-12 \mu \mathrm{m}$ as compared with 3-6.5 $\mu \mathrm{m}$ for haploids; Hastie \& Heale, 1985) was recorded.

\section{RESULTS}

Thirty stable monoauxotrophs were recovered after either UV or NTG mutagenesis, fourteen required arginine, three methionine, three lysine, two tyrosine, two inositol, three nicotinamide, two adenine and one leucine. Heterokaryon compatibility studies using auxotrophs have not usually used monoauxotrophs because of the possibility of reversion to prototrophy and subsequent growth on MM. In this study, the possibility of reversion was decreased by limiting both the period of growth on CM agar required to produce a conidial suspension to $3-4 \mathrm{~d}$, and the incubation time of the mixed conidial suspension in liquid CM to $24 \mathrm{~h}$. After this period, a mycelial pellet was produced that could be washed in sterile distilled water and transferred to GMM. In a number of cases either slight growth of an individual auxotroph on GMM or reversion to prototrophy made it impossible to evaluate compatibility and these auxotrophs were discarded.

Table 2 lists the 90 combinations of complementary auxotrophs tested in this study as well as the number out of 100 mixed mycelial pellets that produced prototrophic heterokaryotic growth after $20 \mathrm{~d}$ incubation on GMM. Thirty crosses produced heterokaryotic growth over this period. All 14 crosses that were fully $(100 \%)$ heterokaryon compatible produced some prototrophic growth by $6 \mathrm{~d}$ and growth from all pellets by $11 \mathrm{~d}$. The majority $(12 / 14)$ of these had produced some heterokaryotic growth after $2 \mathrm{~d}$ incubation on GMM. Prototrophic growth was observed between 5 and $13 \mathrm{~d}$ for the 16 crosses of auxotrophs which were partially $(1-99 \%)$ compatible. In all cases growth of the heterokaryon was slow. Some specific auxotrophic markers, notably 10 tyr- 1 and 4 lys- 1 , appeared to interfere with heterokaryon formation or growth.

Crosses between the North American isolates (2,7 and 8) and Australian isolates (5, 6, 12 and 13) failed to form heterokaryons, but within each of these two groups (with the exception of isolate 5 paired with 13$)$ the isolates were fully $(100 \%)$ heterokaryon compatible (Table 3$)$. In contrast, the three European isolates (3,4 and 10 ) showed variable compatibility with both the North American and Australian isolates. The European isolate 4 was compatible with three Australian isolates $(5,12$ and 13$)$ and two North American isolates $(2,8)$. The European isolate 3 was compatible with two Australian isolates ( 5 and 12) and two North American isolates (2 and 7) as well as both other European isolates. European isolate 10 was only compatible with two Australian isolates (12 and 13) and the other Greek isolate (3). With the possible exception of isolate 10 , pathogenicity (race designation) did not appear to affect compatibility.

All 90 crosses were assessed for the production of heterozygous diploids by sampling five heterokaryons each day for a $20 \mathrm{~d}$ period (Table 4). Diploids were identified as monoconidial isolates showing prototrophic growth and producing conidia in the diploid size range 8-12 $\mu \mathrm{m}$ (Hastie \& Heale, 1985). Heterozygous diploids were recovered from all crosses between North American isolates but not from crosses between the Australian isolates. The yield of diploids was generally low and in most cases they were recovered after at least $10 \mathrm{~d}$ incubation of the 
Table 2. Individual pairs of auxotrophic mutants of $V$. dahliae used in heterokaryon compatibility testing

Compatibility was assessed as the percentage of mixed mycelial pellets producing prototrophic growth after $20 \mathrm{~d}$ incubation on GMM.

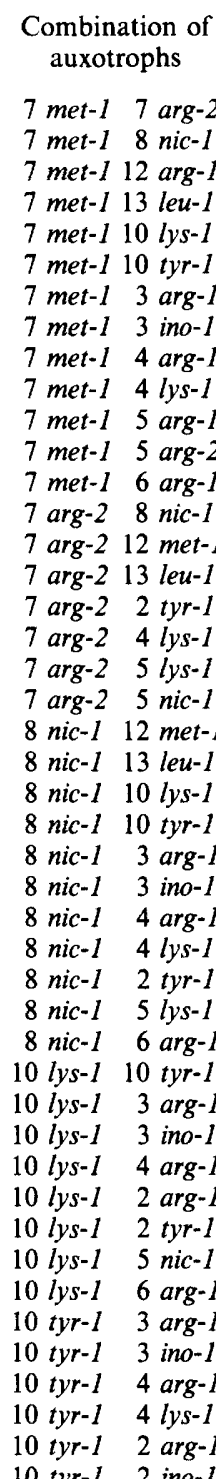

\section{Compatibility}

100

100

0

0

0

10

24

0

0

0
0

100

0

100

0
0

0

0

0
0

0

0

0
23

0

100

0
0

0

26

74

0

0

0
0

0

0

0

0

0

0

$$
\begin{gathered}
\text { Combination of } \\
\text { auxotrophs }
\end{gathered}
$$

10 tyr-1 5 nic-1

10 tyr-1 5 lys-1

10 tyr-1 6 arg-1

12 met-1 13 leu-1

12 met-1 10 lys -1

12 met-1 10 tyr-1

12 met-1 3 arg-1

12 met-1 3 ino- 1

12 met-1 4 arg-1

12 met-1 4 lys-1

12 met-2 2 arg-1

12 met-1 2 tyr-1

12 met-1 5 nic-1

12 met-1 6 arg-1

13 leu-1 10 lys-1

13 leu-1 10 tyr-1

13 leu-1 3 arg-1

13 leu-1 3 ino-1

13 leu-1 4 arg-1

13 leu-1 4 lys-1

13 leu-1 2 arg-1

13 leu-1 2 ino-1

13 leu-1 2 tyr-1

13 leu-1 5 lys-1

13 leu-1 5 nic-1

13 leu-1 6 arg-1

2 arg- 15 nic- 1

2 ino-1 6 arg-1

2 tyr-1 5 lys-1

2 tyr-1 5 nic-1

2 tyr-1 6 arg-1

5 nic-1 6 arg-1

3 ino-1 4 arg-1

3 ino-1 4 lys-1

3 arg-1 4 lys-1

3 ino-1 2 tyr-1

3 ino-1 5 lys- 1

3 ino-1 5 nic-1

3 ino-1 6 arg-1

4 lys-1 2 tyr-1

4 arg-1 2 tyr-1

4 arg-1 5 nic-1

4 arg-1 5 lys-1

4 lys $-1 \quad 4$ arg-1

4 lys-1 6 arg-1
Compatibility

$$
\begin{array}{r}
0 \\
0 \\
0 \\
100 \\
26 \\
0 \\
15 \\
100 \\
20 \\
8 \\
0 \\
0 \\
100 \\
100 \\
18 \\
0 \\
0 \\
0 \\
58 \\
22 \\
0 \\
0 \\
0 \\
0 \\
15 \\
100 \\
0 \\
0 \\
0 \\
0 \\
0 \\
100 \\
25 \\
0 \\
0 \\
100 \\
100 \\
100 \\
0 \\
0 \\
23 \\
28 \\
0 \\
0 \\
0
\end{array}
$$

heterokaryons. Only the 'selfing' 7 met-1/7 arg-2 produced diploids over most of the $20 \mathrm{~d}$ period. Diploids were recovered from only 13 of the 30 crosses that produced heterokaryotic growth. Two crosses ( 7 met-1/10 lys -1 and 7 met $-1 / 6$ arg-1) produced no visible prototrophic growth on GMM but rare diploids were recovered. Ten monoconidial prototrophic diploids from each of the 15 crosses that had produced diploids were subcultured onto CM and the genotypes of the subsequent sectors that emerged were characterized. All diploids produced both prototrophic and auxotrophic sectors. For the majority of diploids only one of the parental auxotrophic markers was recovered. Both parental markers were recovered from at least one diploid for nine crosses. 
Table 3. Mean compatibility for pairings of auxotrophic mutants derived from wild-type isolates of $V$. dahliae

Compatibility is expressed as the mean number of heterokaryons produced between pairs of auxotrophs after $20 \mathrm{~d}$ incubation on GMM.

\begin{tabular}{|c|c|c|c|c|c|c|c|c|c|c|}
\hline \multirow{3}{*}{$\begin{array}{c}\text { Auxotrophic } \\
\text { mutant }\end{array}$} & \multicolumn{10}{|c|}{ Compatibility } \\
\hline & \multicolumn{10}{|c|}{ Auxotrophic mutant: } \\
\hline & 2 & 7 & 8 & 5 & 6 & 12 & 13 & 10 & 3 & 4 \\
\hline 2 & NT & 100 & 100 & 0 & 0 & 0 & 0 & 0 & 100 & $11 \cdot 5$ \\
\hline 7 & & 100 & 100 & 0 & $0^{*}$ & 0 & 0 & $0^{*}$ & 17 & 0 \\
\hline 8 & & & NT & 0 & 0 & 0 & 0 & 0 & 0 & $11 \cdot 5$ \\
\hline 5 & & & & NT & 100 & 100 & $7 \cdot 5$ & 0 & 100 & 14 \\
\hline 6 & & & & & NT & 100 & 100 & 0 & 0 & 0 \\
\hline 12 & & & & & & $\mathrm{NT}$ & 100 & 13 & 57.5 & 14 \\
\hline 13 & & & & & & & NT & 9 & 0 & 40 \\
\hline 10 & & & & & & & & 0 & 50 & 0 \\
\hline 3 & & & & & & & & & NT & $8 \cdot 3$ \\
\hline 4 & & & & & & & & & & 0 \\
\hline
\end{tabular}

NT, Not tested.

* Rare diploids recovered.

\section{DISCUSSION}

Although only 10 wild-type isolates of $V$. dahliae were used in this study, two heterokaryon incompatibility groups could be identified. Isolates within one group formed heterokaryons with each other but not with members of the other group. One group contained the three North American isolates and the other group the four Australian isolates. Puhalla (1979) suggested that heterokaryon incompatibility in $V$. dahliae may indicate genetic divergence between populations. If these isolates of $V$. dahliae did share a common origin, geographical isolation may have caused the Australian and North American groups to diverge to an extent that they can no longer interact to form heterokaryons. Using auxotrophs and microsclerotial colour mutants, Puhalla (1979) classified 19 isolates of $V$. dahliae from 15 different hosts and geographical origins into four heterokaryon incompatibility groups. The number of isolates was extended to 94 representing 38 different host plants and these were classified into 16 distinct heterokaryon incompatibility groups and a number of non-reactors (Puhalla \& Hummel, 1983). In the present study the European isolates 4 and 10 were partially compatible with two members (isolates 13 and 14) of the Australian groups. European isolates 3 and 4 were also partially compatible with the North American isolates 7 and 8 respectively. It is interesting to note that in the studies of Puhalla (1979) and Puhalla \& Hummel (1983) Australian and North American isolates were rarely heterokaryon compatible whereas European isolates were generally compatible with either the North American or Australian isolates. Together with the work presented here, this may suggest that European isolates of $V$. dahliae are heterogeneous, some showing genetic homology with both North American and Australian isolates. This could be investigated further using more isolates.

Caten \& Jinks (1966) have criticized the use of auxotrophs for heterokaryon compatibility studies because of the strong selective pressure imposed and have advocated the use of nonselective or neutral genetic markers such as spore colour mutants. More recent studies with $\boldsymbol{A}$. nidulans using both auxotrophic and spore colour mutants have suggested that the classification of isolates into heterokaryon incompatibility groups is not strongly influenced by the markers used (Dales et al., 1983). Similarly, Puhalla \& Hummel (1983) found that the crosses of complementary diauxotrophs of $V$. dahliae that produced prototrophic heterokaryotic growth on $\mathrm{MM}$ were the same crosses that produced heterokaryons when microsclerotial colour mutants were used. The failure, in this study, of 'two selfing' (10 tyr- $1 \times 10 \mathrm{lys}-1$ and $4 \mathrm{lys}-1 \times 4 \mathrm{arg}-1$ ) to establish heterokaryons may be attributable to the pleiotropic effect of specific auxotrophic markers. 
Table 4. Combination of auxotrophs of $V$. dahliae from which heterozygous diploids were obtained and their respective frequency of recovery over a 20 d period

Five mixed mycelial pellets (heterokaryons) from each pair of auxotrophs were sampled each day over a $20 \mathrm{~d}$ period by washing in $5 \mathrm{ml}$ sterile distilled water and spreading the spore suspension onto MM. Diploid colonies were identified by conidial size measurements.

\begin{tabular}{|c|c|c|c|c|c|}
\hline \multirow[b]{3}{*}{$\begin{array}{l}\text { No. of } \\
\text { days }\end{array}$} & \multicolumn{5}{|c|}{ Frequency of recovery } \\
\hline & \multicolumn{5}{|c|}{ Combination of auxotrophs: } \\
\hline & $\begin{array}{c}7 \text { met }-11 \\
7 \text { arg- } 2\end{array}$ & $\begin{array}{c}7 \text { met }-11 \\
8 \text { nic }-1\end{array}$ & $\begin{array}{l}7 \text { met }-1 / \\
10 \text { lys }-1\end{array}$ & $\begin{array}{c}7 \text { met }-11 \\
3 \text { ino- } 1\end{array}$ & $\begin{array}{c}7 \text { met }-11 \\
6 \text { arg- } 1\end{array}$ \\
\hline 1 & 0 & 0 & 0 & $\mathbf{0}$ & 0 \\
\hline 2 & 0 & 0 & 0 & 0 & 0 \\
\hline 3 & 0 & 0 & 0 & 0 & 0 \\
\hline 4 & 0 & 0 & 0 & 0 & 0 \\
\hline 5 & 30 & 0 & 0 & 0 & 0 \\
\hline 6 & 250 & 0 & $\mathbf{0}$ & 0 & 0 \\
\hline 7 & 100 & 0 & 0 & 0 & 0 \\
\hline 8 & 250 & 0 & 0 & 0 & 0 \\
\hline 9 & 200 & 0 & 0 & 0 & 0 \\
\hline 10 & 340 & 0 & 0 & 157 & 0 \\
\hline 11 & 250 & 0 & 0 & 41 & 0 \\
\hline 12 & 200 & 0 & 0 & 19 & 0 \\
\hline 13 & 212 & 0 & 0 & 30 & 0 \\
\hline 14 & 180 & 35 & 0 & 128 & 0 \\
\hline 15 & 102 & 90 & 0 & 80 & 0 \\
\hline 16 & 40 & 190 & 28 & 21 & 33 \\
\hline 17 & 30 & 182 & 0 & 29 & 0 \\
\hline 18 & - & 0 & 0 & - & 0 \\
\hline 19 & 50 & 0 & 0 & 23 & 0 \\
\hline \multirow[t]{2}{*}{20} & 90 & 0 & 0 & - & 20 \\
\hline & $\begin{array}{l}7 \text { arg- } 11 \\
2 \text { tyr-I }\end{array}$ & $\begin{array}{l}7 \text { arg- } 1 / \\
8 \text { nic-1 }\end{array}$ & $\begin{array}{l}8 \text { nic-1/ } \\
2 \text { tyr-1 }\end{array}$ & $\begin{array}{l}8 \text { nic-1/ } \\
4 \text { arg-1 }\end{array}$ & $\begin{array}{c}12 \text { met }-1 / \\
4 \text { arg-I }\end{array}$ \\
\hline 1 & 0 & 0 & 0 & 0 & 0 \\
\hline 2 & 0 & 0 & 0 & 0 & 0 \\
\hline 3 & 0 & 0 & 0 & 0 & 0 \\
\hline 4 & 0 & 0 & 0 & 0 & 0 \\
\hline 5 & 0 & 0 & 0 & 0 & 0 \\
\hline 6 & 0 & 0 & 0 & 0 & 0 \\
\hline 7 & 47 & 0 & 0 & 0 & 0 \\
\hline 8 & 49 & 0 & 0 & 0 & 0 \\
\hline 9 & 55 & 0 & 0 & 0 & 0 \\
\hline 10 & 58 & 0 & 0 & 0 & 0 \\
\hline 11 & 62 & 0 & 0 & 0 & 0 \\
\hline 12 & 70 & 0 & 0 & 0 & 0 \\
\hline 13 & 73 & 0 & 0 & 60 & 0 \\
\hline 14 & 0 & 0 & 0 & 70 & 0 \\
\hline 15 & 0 & 26 & 0 & 0 & 0 \\
\hline 16 & 0 & 196 & 10 & 0 & 0 \\
\hline 17 & 0 & 10 & 15 & 0 & 20 \\
\hline 18 & 0 & 0 & 30 & 0 & 160 \\
\hline 19 & 0 & 0 & 300 & 0 & 0 \\
\hline 20 & 0 & 0 & 0 & 0 & 0 \\
\hline
\end{tabular}

In this study, the frequency of heterozygous diploid formation was measured as the number of diploids recovered from five randomly selected heterokaryons sampled each day over a $20 \mathrm{~d}$ period. Diploids were only recovered from 15 of the 90 crosses. With the exception of crosses between Australian isolates, diploids were generally recovered at a higher rate from fully heterokaryon compatible pairings. This is not surprising as heterokaryon formation precedes the formation of heterozygous diploids. Fully compatible, and therefore perhaps genetically 
Table 4. (continued)

\begin{tabular}{|c|c|c|c|c|c|}
\hline \multirow[b]{3}{*}{$\begin{array}{l}\text { No. of } \\
\text { days }\end{array}$} & \multicolumn{5}{|c|}{ Frequency of recovery } \\
\hline & \multicolumn{5}{|c|}{ Combination of auxotrophs: } \\
\hline & $\begin{array}{c}13 \text { leu- } 11 \\
4 \text { arg- } 1\end{array}$ & $\begin{array}{l}13 \text { leu-1/ } \\
10 \text { lys }-1\end{array}$ & $\begin{array}{c}10 \text { lys }-1 / \\
3 \text { ino-1 }\end{array}$ & $\begin{array}{l}3 \text { ino- } 11 \\
5 \text { nic- } 1\end{array}$ & $\begin{array}{c}4 \text { arg- } 1 / \\
2 \text { tyr-1 }\end{array}$ \\
\hline 1 & 0 & 0 & 0 & 0 & 0 \\
\hline 2 & 0 & 0 & 0 & 0 & 0 \\
\hline 3 & 0 & 0 & 0 & 0 & 0 \\
\hline 4 & 0 & 0 & 0 & 0 & 0 \\
\hline 5 & 0 & 0 & 0 & 0 & 0 \\
\hline 6 & 0 & 0 & 0 & 0 & 0 \\
\hline 7 & 0 & 0 & 0 & 0 & 0 \\
\hline 8 & 0 & 0 & 0 & 0 & 0 \\
\hline 9 & 0 & 0 & 0 & 0 & 0 \\
\hline 10 & 142 & 0 & 0 & 0 & 0 \\
\hline 11 & 0 & 52 & 0 & 0 & 0 \\
\hline 12 & 0 & 0 & 0 & 0 & 0 \\
\hline 13 & 0 & 0 & 15 & 0 & 0 \\
\hline 14 & 0 & 0 & 20 & 26 & 30 \\
\hline 15 & 0 & 0 & 30 & 196 & 128 \\
\hline 16 & 0 & 0 & 0 & 10 & 80 \\
\hline 17 & 0 & 0 & 0 & 0 & 0 \\
\hline 18 & 0 & 0 & 0 & 0 & 0 \\
\hline 19 & 0 & 0 & 0 & 0 & 0 \\
\hline 20 & 0 & 0 & 0 & 0 & 0 \\
\hline
\end{tabular}

similar, isolates are also perhaps more likely to produce heterozygous diploids because of a greater synchrony of cell cycles (Ingle \& Hastie, 1974). Although heterokaryons were not detected for the crosses 7 met-1/10 lys-1 and 7 met-1/6 arg-1, rare heterozygous diploids were recovered, presumably indicating the presence of at least a transient heterokaryotic stage. Although the Australian isolates used in this study belonged to the same compatibility group, no diploids were recovered from these crosses. Possibly the conditions used in this study were unsuitable for diploidization in these isolates. It should be noted, however, that one of the two pairings that produced diploids despite being rated as zero compatibility, involved an Australian isolate.

Interestingly, where heterozygous diploids were produced, they were harvested either on one day only or on consecutive days during the $20 \mathrm{~d}$ period (Table 4). This was apparent usually as a discrete period of diploid production occurring before the end of the $20 \mathrm{~d}$ period. The reasons for this are unclear, but may suggest that for each cross, there is an optimum time for diploid production, but that once produced, diploid conidia are either viable for only a few days or germinate to produce a haploid or aneuploid colony. It is also possible that colonies producing diploid conidia arose from diploid hyphal fragments, rather than conidia, removed from the heterokaryon but that diploid hyphae which had not been transferred reverted to a haploid state before producing conidia.

This study has demonstrated the existence of different levels of vegetative or heterokaryon incompatibility between isolates of $V$. dahliae pathogenic towards tomato. Heterozygous diploids were recovered from crosses between isolates differing in pathogenicity (race) and geographical origin indicating the potential for the genetic analysis of pathogenicity through the parasexual cycle.

\section{REFERENCES}

CATEN, C. E. \& JiNKs, J. L. (1966). Heterokaryosis: its significance in wild homothallic Ascomycetes and fungi imperfecti. Transactions of the British Mycological Society 49, 81-93.
Clarkson, J. M. \& Heale, J. B. (1985a). Pathogenicity and colonization studies on wild-type and auxotrophic isolates of Verticillium albo-atrum from hop. Plant Pathology 34, 119-128. 
Clarkson, J. M. \& Heale, J. B. (1985b). Heterokaryon compatibility and genetic recombination within a host plant between hop wilt isolates of Verticillium albo-atrum from hop. Plant Pathology 34, 129138.

Dales, R. B., Moorhouse, J. \& Croft, J. M. (1983). The location and analysis of two heterokaryon incompatibility loci in strains of Aspergillus nidulans. Journal of General Microbiology 129, 3637-3642.

Demerec, M., Adelberg, E. A., Clark, A. J. \& Hartman, P. E. (1966). A proposal for a uniform nomenclature in bacterial genetics. Genetics 54, 6176.

Fordyce, C. \& GreEN, R. J. (1964). Mechanisms of variation in Verticillium dahliae. Phytopathology 54, 795-798.

HASTIE, A. C. (1962). Genetic recombination in the hop wilt fungus Verticillium albo-atrum. Journal of General Microbiology 27, 373-382.

Hastie, A. C. (1973). Hybridization of Verticillium albo-atrum and Verticillium dahliae. Transactions of the British Mycological Society 60, 511-523.

Hastie, A. C. \& Heale, J. B. (1985). Genetics of Verticillium. In Phytopathologia Mediterranea, pp. 130-163. Italy: Unione Fitopatologica Mediterranea.

HEALE, J. B. (1966). Heterokaryon synthesis and morphogenesis in Verticillium. Journal of General Microbiology 45, 419-427.

Heale, J. B. (1988). Verticillium spp., the cause of vascular wilts in many species. Advances in Plant Pathology 6, 291-312.

INGLE, M. R. \& HASTIE, A. C. (1974). Environmental factors affecting the formation of diploids in Verticillium albo-atrum. Transactions of the British Mycological Society 62, 313-321.

Jinks, J. L., CATEN, C. E., Simchen, G. \& Croft, J. H. (1966). Heterokaryon incompatibility and variation in wild populations of Aspergillus nidulans. Heredity 21, 227-239.
Mace, M. E., Bell, A. A. \& Beckman, C. H. (1981). Fungal Wilt Diseases of Plants. New York: Academic Press.

O'Garro, L. W. \& Clarkson, J. M. (1988). Pathogenicity of race-1 and race-2 tomato wilt isolates of Verticillium dahliae from different geographical origins. Journal of Phytopathology (in the Press).

PegG, G. F. (1974). Verticillium diseases. Review of Plant Pathology 53, 157-182.

Perkins, D. D. \& BARRY, E. G. (1977). The cytogenics of Neurospora. Advances in Genetics 19, 133-285.

Puhalla, J. E. (1979). Classification of isolates of Verticillium dahliae based on heterokaryon incompatibility. Phytopathology 69, 1186-1189.

PuHalla, J. E. (1984a). A visual indicator of heterokaryosis in Fusarium oxysporum from celery. Canadian Journal of Botany 62, 540-545.

Puhalla, J. E. (1984b). Races of Fusarium oxysporum f. sp. apii in California and their genetic interrelationships. Canadian Journal of Botany 62, 546550.

Puhalla, J. E. (1985). Classification of strains of Fusarium oxysporum on the basis of vegetative compatibility. Canadian Journal of Botany 63, 179183.

Puhalla, J. E. \& Hummel, M. (1983). Vegetative compatibility groups within Verticillium dahliae. Phytopathology 73, 1305-1308.

Puhalla, J. E. \& Speith, P. T. (1983). Heterokaryosis in Fusarium moniliforme. Experimental Mycology 7, 328-335.

TyPas, M. A. \& Heale, J. B. (1976). Heterokaryosis and the role of cytoplasmic inheritance in dark resting structure formation in Verticillium spp. Molecular and General Genetics 146, 17-26.

Typas, M. A. \& Heale, J. B. (1978). Heterozygous diploid analyses via the parasexual cycle and a cytoplasmic pattern of inheritance in Verticillium spp. Genetical Research 31, 131-144. 